\title{
NueVAS EVIDENCIAS HISTÓRICAS DEL SIGLO XVIII SOBRE LA PRESENCIA DE "CARDOS" EN ARgENTINA Y SUS IMPLICANCIAS ETNOBOTÁNICAS
}

\author{
NeW HISTORICAL EVIDENCES OF THE 18TH CENTURY ON PRESENCE OF \\ "THISTLES" IN ARGENTINA AND ITS ETHNOBOTANICAL IMPLICATIONS
}

\author{
Diego G. Gutiérrez ${ }^{1,2}$, Gustavo F. Scarpa ${ }^{1}\left[\right.$ y Cintia N. Rosso' ${ }^{10}$
}

\begin{abstract}
1. División Plantas Vasculares, Museo Argentino de Ciencias Naturales, Consejo Nacional de Investigaciones Científicas y Técnicas, Av. Ángel Gallardo 470, C1405DJR, ciudad de Buenos Aires, Argentina.

2. Laboratorio de Morfología Comparada de Espermatófitas, Facultad de Ciencias Agrarias y Forestales, Universidad Nacional de La Plata, La Plata, Buenos Aires, Argentina.

*ddigutier@macn.gov.ar

Citar este artículo GUTIÉRREZ, D. G., G. F. SCARPA y C. N. ROSSO. 2020. Nuevas evidencias históricas del siglo XVIII sobre la presencia de "cardos" en Argentina y sus implicancias etnobotánicas. Bol. Soc. Argent. Bot. 55: 295-310.
\end{abstract}

DOI: https://doi. org/10.31055/1851.2372.v55. n2.26407

Recibido: 13 Noviembre 2019

Aceptado: 28 Mayo 2020

Publicado: 30 Junio 2020

Editora: Norma Hilgert iD

ISSN versión impresa 0373-580X

ISSN versión on-line 1851-2372

\section{SUMMARY}

Background and aims: Currently, many species called "thistles" are widely distributed in Argentina. During 18th Century several Jesuit publications mentioned "thistles" as the work made by Florian Paucke. The goal of this work is to gather, from writing sources of the 18th Century, plant records called as "thistles" or similar names; to identify at species level the plant entities mentioned by Paucke as "azafrán", "cardo", and "cardo hortense"; and to discuss ethnobotanical implications.

M\&M: The study area included Argentina from the Pampean region to the Chacoan region. Historical evidences of the $18^{\text {th }}$ Century, herbarium materials and their morphology were analyzed, and field trips were made.

Results: Entities "azafrán alazor", "de Indias" o "paracuario" by Paucke were identified as Carthamus tinctorius ("safflower"), and "cardo" and "cardo hortense" as Cynara cardunculus (Compositae), associated with varieties sylvestris ("thistle") and scolymus ("artichoke"), respectively. "Cardo santo" named by Pedro de Montenegro was associated to Argemone subfusiformis (Papaveraceae). Twelves new medicinal and food uses of the Moqoit from the Chaco of Argentina were recorded.

Conclusions: Identified "thistles" were used for medicinal and nutritional purposes during the colonial period, and incorporated at aboriginal missions by Jesuit priests. Carthamus tinctorius and Cynara cardunculus may have been introduced in orchards and later on have escaped of the cultivation areas.

\section{KEY WORDS}

Artichoke, Carthamus, Cynara, ethnobotany, Paucke, safflower, thistle.

\section{RESUMEN}

Introducción y objetivos: Actualmente numerosas especies llamadas "cardos" están ampliamente distribuidas en Argentina. Durante el siglo XVIII varias publicaciones jesuíticas mencionaron "cardos", como el trabajo realizado por Florián Paucke. EI objetivo del trabajo es recopilar, de las fuentes escritas del siglo XVIII, los registros de plantas denominadas como "cardos" o nombres similares; identificar a nivel de especie las entidades mencionadas por Paucke como "azafrán", "cardo" y "cardo hortense"; y discutir las implicancias etnobotánicas.

M\&M: El área de estudio incluyó Argentina desde la región Pampeana a la región Chaqueña. Se analizaron evidencias históricas del siglo XVIII, materiales de herbario y morfología, y se efectuaron viajes de campo.

Resultados: Las entidades "azafrán alazor", "de Indias" o "paracuario" de Paucke se identificaron como Carthamus tinctorius ("cártamo"), y "cardo" y "cardo hortense" como Cynara cardunculus (Compositae), asociadas a las variedades sylvestris ("cardo de Castilla") y scolymus ("alcaucil") respectivamente. El "cardo santo" nombrado por Pedro de Montenegro se asoció a Argemone subfusiformis (Papaveraceae). Se registraron 12 nuevos usos medicinales y alimenticios de los moqoit del Chaco de Argentina.

Conclusiones: Los "cardos" identificados fueron utilizados con fines medicinales y alimenticios durante el período colonial, e incorporados en las reducciones aborígenes por misioneros jesuitas. Carthamus tinctorius y Cynara cardunculus posiblemente hayan sido introducidos en huertos y posteriormente hayan escapado de las áreas de cultivo.

\section{Palabras Clave}

Alcaucil, cardo, cártamo, Carthamus, Cynara, etnobotánica, Paucke. 


\section{INTRODUCCIÓN}

La introducción de especies exóticas en las colonias europeas de América implicó, entre otras cosas, el intercambio de plantas, saberes y prácticas, pudiéndose definir este proceso como la importación de un modo de vida occidental al Nuevo Mundo (Gruzinski, 2007). Los europeos buscaban reproducir el modelo occidental en tierras americanas a partir de la introducción de sus propios bienes culturales y naturales domesticados (plantas $\mathrm{y}$ animales) y de la modificación concomitante de los sistemas religiosos y económicos aborígenes. Es así como se construyeron jardines y huertos en áreas controladas de las colonias y las misiones donde la mera práctica agrícola, por ejemplo, representaba "civilizar" el espacio salvaje de América (Rosso, 2012). Este proceso de introducción de especies vegetales en cada región del continente adquirió características singulares, dependiendo de la zona considerada y de los actores sociales y plantas involucradas en cada caso.

En América del Sur una cantidad importante de especies de plantas introducidas por los españoles $\mathrm{y}$ portugueses se incorporaron rápidamente a la alimentación de los pueblos originarios del Nuevo Mundo como el "trigo" (Triticum sp., Poaceae), la "cebada" (Hordeum sp., Poaceae), los "duraznos" (Prunus sp., Rosaceae) y los "cítricos" (Citrus sp., Rutaceae) (Capparelli et al., 2005; Hilgert et al., 2014; Stampella, 2015). Los "cardos" dado su uso en el Viejo Mundo formaron parte de este intercambio (Hernández Bermejo et al., 2019). El término "cardo" se utiliza frecuentemente en Iberoamérica, histórica y actualmente, para designar a plantas herbáceas espinosas pertenecientes a especies de Compositae de la tribu Cardueae (Font Quer, 1993). Dicho término proviene del latín "arduus" y del celta "ard" indicando la presencia de espinas (e.g. Ariza Espinar \& Delucchi, 1998). Cardueae presenta unas 2400 especies, principalmente nativas de la región Mediterránea del Viejo Mundo (Susanna \& García-Jacas, 2007, 2009), siendo muchas de ellas utilizadas desde antaño en el arte, la alimentación y la medicina: e.g. Carthamus tinctorius L., Centaurea benedicta (L.) L., Cynara cardunculus L. (Rivera \& Obón, 1996; Hernández Bermejo et al., 2019). Por este motivo, la distribución de muchas de sus especies ha sido influenciada por el comercio o la agricultura (e.g. Centaurea cyanus L. y Onopordum tauricum Willd.) y actualmente algunas de ellas forman parte de los principales cultivos mundiales, como es el caso del "alcaucil" y el "cártamo" (Ekin, 2005; Simpson, 2009; Calabrese, 2016). En América del Sur, Cardueae está presente en casi todos sus países desde Colombia y Venezuela hasta Argentina y Chile y se encuentra representada por más de 35 especies entre nativas y exóticas introducidas (Dillon \& Hensold, 1993; Robinson et al., 1999; Badillo, 2001; Dillon, 2006; Delucchi, 2008; Beck et al., 2014; Delucchi \& Gutiérrez, 2014; Ávila et al., 2016; Cordero et al., 2016). De estas especies, la gran mayoría (i.e. 26, aquellas pertenecientes a los géneros Arctium L., Carduus L., Carthamus L., Centaurea L., Cirsium Mill., Cynara L., Onopordum L., Rhaponticum Vaill. y Silybum Vaill.) son malezas desde el punto de vista agronómico de ambientes templados y mediterráneos especialmente, predominando en la región Pampeana de Argentina y Uruguay y en el centro de Chile (Marzocca 1986; Katinas et al., 2007; Delucchi, 2008; Delucchi \& Gutiérrez, 2014; Fernández et al., 2016).

En el caso particular de Argentina, hasta la actualidad, con el nombre común "cardo" y palabras relacionadas (e.g. "carda", "cardencha", "cardón”) se han registrado 16 especies de Cardueae (Schulz, 1976; Correa et al., 2003; Peña \& Pensiero, 2004; Rosso \& Scarpa, 2012). Muchas de éstas se encuentran naturalizadas y poseen registros de sus diversos usos populares pasados y presentes (e.g. Azara, 1809; Hieronymus, 1882; Toursarkissian, 1980; Roig, 2001; Rosso \& Scarpa, 2012) e incluso forman parte de la actividad agroindustrial actual (Gominho et al., 2009; Calabrese, 2016; Delucchi et al., 2017; Hernández Bermejo et al., 2019). Asimismo, bajo esta denominación se pueden encontrar también otras 20 especies provenientes de Angiospermas de diferentes grupos taxonómicos y orígenes geográficos (Correa et al., 2003; Peña \& Pensiero, 2004). Por lo general estas últimas se corresponden con especies que presentan órganos espinosos y/o agrupamientos de sus flores en inflorescencias similares en aspecto a un capítulo: por ejemplo, Apiaceae (i.e. Eryngium sp.), Bromeliaceae (i.e. Aechmea sp., Bromelia sp.), Cactaceae (i.e. Cereus sp., Trichocereus sp.), Calyceraceae (i.e. Acicarpha sp.), Caprifoliaceae (i.e. Dipsacus sp.), Papaveraceae (i.e. Argemone 


\section{G. Gutiérrez et al. - "Cardos" en Argentina en el siglo XVIII y etnobotánica}

sp.) (Correa et al., 2003; Barboza et al., 2006). Las causas de la llegada de los "cardos" y especies afines exóticos (i.e., Arctium, Carduus, Carthamus, Centaurea, Cirsium, Cynara, Onopordum, Rhaponticum y Silybum) al sur de América del Sur podrían deberse a su utilización desde los siglos XVI y XVII por los exploradores europeos $\mathrm{y}$ colonos en la alimentación y como cultivos en jardines de los primeros asentamientos, así como de forma involuntaria como maleza (Hernández Bermejo et al., 2019 y referencias allí citadas). Por otro lado, para la región pampeana se ha propuesto que algunas especies de "cardos" habrían sido introducidas de manera accidental a través del transporte de semillas de trigo; en particular para las especies Cynara cardunculus ("cardo de Castilla") y Silybum marianum (L.) Gaertn. ("cardo mariano") en los alrededores de Buenos Aires (Amaral, 1997, 1999; Recalt, 2004). En la misma línea explicativa se ha sostenido que la expansión de los "cardales" habría sido facilitada por disturbios antropogénicos en las pampas debido a la actividad del ganado vacuno y equino desde mediados del siglo XVI (Delucchi et al., 2003; Soria, 2012). Sin embargo, no hay datos fidedignos para poder identificar las entidades mencionadas como "cardos" a nivel taxonómico que han estado involucradas en dicho proceso.

La alusión a "cardos" exóticos resulta indiscutible en numerosas narraciones de naturalistas y exploradores de principios del XIX (e.g., Azara, 1809; Darwin, 1839; Head, 1846). En el siglo XIX las referencias de "cardos" en especial en floras y listas de plantas son precisas al nivel de especies y están acompañadas en muchos casos con descripciones morfológicas detalladas y especímenes conservados en herbarios. Por ejemplo, Carthamus tinctorius, Centaurea benedicta, $C$. calcitrapa L., C. melitensis L., Cynara cardunculus y Silybum marianum se describen principalmente para el centro de Chile y las pampas de Argentina y Uruguay (e.g., Hooker \& Arnott, 1835; Rémy, 1849; Berg, 1877; Grisebach, 1879; Baker, 1884).

En contraposición, nuestro conocimiento sobre los "cardos" en el sur de América del Sur previamente al siglo XIX resulta escaso e impreciso. Como ya fuera mencionado por Delucchi et al. (2003), las primeras pruebas sobre la presencia de estas plantas, nativas o introducidas, en la región halladas en fuentes escritas serían las de Cardiel
([1748] 1930) y Concolorcorvo ([1773] 1908). Ambos autores refieren la existencia de "cardales" utilizados como leña, a $400 \mathrm{~km}$ de la ciudad de Buenos Aires y alrededores, entre los años 1748 y 1749 , aunque su identificación taxonómica resulta incierta. Una excepción a ello la constituye la referencia del jesuita Florián Paucke ([1776-1778] 1944) en las memorias de su estadía desde 1749 a 1767 entre los aborígenes mocovíes de San Javier, en el centro-este de Argentina, citado por Rosso \& Scarpa (2012). El trabajo de este misionero resulta un punto de partida fundamental para comprender la presencia y utilización de los "cardos" por parte de las poblaciones nativas sudamericanas y colonos debido a que se encuentran descriptos en el texto y representados iconográficamente con gran detalle en láminas realizadas por él mismo (Zanetti, 2013). Entre las entidades mencionadas y/o ilustradas por Paucke, tres corresponderían a taxa pertenecientes a Cardueae. Una de dichas identidades, denominada "azafrán alazor", “azafrán de Indias" o "azafrán paracuario", fue asociada a las especies Carthamus lanatus L. y C. tinctorius (Wernicke, 1944) y más recientemente a la primera (Rosso \& Scarpa, 2012), cuyo nombre vulgar es "cardo lanudo" por lo cual se la incluye también en el presente estudio. Las características morfológicas de $C$. lanatus no se ajustan completamente a lo observado en la ilustración de Paucke y las dos entidades restantes referidas como "cardo" y "cardo hortense" no pudieron ser asignadas hasta el momento con ninguna especie taxonómica en particular, sino a la familia Compositae en general (Wernicke, 1944; Rosso \& Scarpa, 2012).

El objetivo principal del trabajo es recopilar de fuentes escritas del siglo XVIII de Argentina las citas de plantas que se puedan corresponder con aquellas denominadas como "cardos" o afines; en segundo lugar, identificar a nivel de especie las entidades mencionadas e ilustradas por Paucke como "azafrán” (“azafrán alazor”, “azafrán de Indias" o "azafrán paracuario"), "cardo" y "cardo hortense"; y finalmente, discutir sus implicancias etnobotánicas.

\section{Materiales y Métodos}

El área de estudio del trabajo abarcó las provincias político-administrativas actuales 
argentinas de Buenos Aires, Chaco, Córdoba, Corrientes, Entre Ríos y Santa Fe, más la ciudad autónoma de Buenos Aires. Dicha área de estudio se corresponde en general con los ambientes templados y cálidos del centro, centro-este y norte de Argentina, y desde el punto de vista biogeográfico con las regiones Chaqueña, del Espinal y Pampeana y, finalmente, la vegetación ribereña de los ríos Paraná, Uruguay y del estuario del Plata, asociada a la región Paranaense (Cabrera \& Willink, 1980; Oyarzabal et al., 2018).

En primera instancia se procedió a compilar evidencias históricas escritas representativas del área de estudio donde se pudieran hallar citas de la presencia de "cardos" durante el siglo XVIII dadas las características de las obras o referencias de trabajos previos (e.g. Correa et al., 2003; Delucchi et al., 2003; Rosso \& Scarpa, 2012). Luego se procedió a analizar en detalle las identidades botánicas de dichas citas de "cardos" en función de las características de las menciones escritas y/o ilustraciones. La fuente histórica principal consultada fue la obra del misionero jesuita Florián Paucke, quien vivió en el actual territorio argentino entre los años 1749 y 1767, fechada entre 1776-78 y titulada "Hacia aquí y para allá. Una estadía entre los indios mocobíes" (Paucke, [1776-78] 1942-1944). Esta publicación narra en detalle las percepciones, historia y avatares que este religioso vivenció en la reducción de aborígenes moqoit de San Javier, provincia de Santa Fe (Argentina) durante mediados del siglo XVIII. La gran profusión de detalles registrados en su obra, tanto en la información suministrada como en los dibujos de plantas y animales de sus láminas policromadas, lo convierte en un documento etnográfico de gran valor histórico. Las otras fuentes escritas consultadas, representativas para el área, fueron las obras de los también jesuitas Pedro de Montenegro ([1710] 2007), Pedro Lozano ([1733] 1874), José Cardiel ([1748] 1930), José Sánchez Labrador ([17711776] 1901), Alonso Carrió de la Vandera (bajo el seudónimo Concolorcorvo; [1773] 1908) y Martín Dobrizhoffer ([1784] 1967). Las obras de Montenegro y Sánchez Labrador corresponden a las misiones jesuíticas de la región chaqueña y litoral de Argentina, más aquellas del este de Bolivia (Chiquitanía), Paraguay, sudeste del Brasil y noroeste de Uruguay, aportando información sobre herbolarios y su utilización médica. El trabajo de Lozano abarca el centro y norte del Virreinato del Río de la Plata, incluyendo información de las áreas rioplatense y chaqueña, en relación al área de estudio, alcanzando las provincias actuales argentinas de Santiago del Estero y Tucumán y el Paraguay. Por su lado, Cardiel brinda datos botánicos de las misiones de los guaraníes y colegios jesuitas de las provincias de Corrientes, Santa Fe y Buenos Aires, e incluso de Patagonia y el Paraguay. El escrito de Concolorcorvo, aporta datos de la región rioplatense hasta el Perú. Finalmente, la obra de Dobrizhoffer permite obtener información etnobotánica misionera del área chaqueña de la provincia de Santa Fe en contacto con los extintos aborígenes abipones. Dichas obras se consideran una muestra representativa para el área de estudio en el período analizado, no descartándose la existencia de otros trabajos no estudiados aquí y que pudieran sumar datos novedosos en el futuro.

En el caso particular del trabajo de Paucke, se confrontaron las ilustraciones y descripciones escritas con las especies que actualmente reciben el nombre vulgar de "cardos" en Argentina (e.g. Correa et al., 2003; Peña \& Pensiero, 2004) mediante un análisis morfológico comparado, identificándose caracteres morfológicos macroscópicos vegetativos y reproductivos para reconocer las especies. Asimismo, se analizó la morfología por medio de especímenes provenientes de los herbarios BA, BAA, BAB, BAF, CORD, CTES, LP, LPAG, MERL, SI y UNR los cuales presentan importantes colecciones del área de estudio. Se realizaron viajes de campo en Argentina entre los años 2010 y 2020 a las provincias de Buenos Aires, Chaco, Córdoba, Corrientes, Entre Ríos y Santa Fe, y los especímenes coleccionados fueron depositados en BA.

Para la identificación, caracterización y taxonomía de las especies de Cardueae en Argentina se sigue a Delucchi \& Gutiérrez (2014). En particular para la taxonomía, muy controvertida, de Cynara cardunculus L. y sus categorías infraespecíficas se siguen los trabajos más recientes que proponen tres variedades: $C$. cardunculus var. altilis DC., C. cardunculus var. scolymus (L.) Fiori y C. cardunculus var. sylvestris (Lam.) Fiori. (e.g. Robba et al., 2005; Sonnante et al., 2007; Gatto et al., 2013). 


\section{G. Gutiérrez et al. - "Cardos" en Argentina en el siglo XVIII y etnobotánica}

\section{Resultados}

\section{Nuevas evidencias históricas}

En la obra atribuida al jesuita Pedro de Montenegro denominada "Materia Médica Misionera" se refieren las siguientes aplicaciones medicinales: "La parida que coma cardo, sea cocido, ó el agua de su cocimiento le aumentará la leche. Cardo santo, su cocimiento bebido vale á los dolores gravisimos / mos, y baidos de cabeza, $y$ restituye la memoria perdida." (Montenegro, [1710] 2007: 301). A pesar de la ausencia de descripción o ilustración de la planta referida como "cardo santo" proponemos sobre la base del nombre vulgar y sus aplicaciones medicinales que se trataría de Argemone subfusiformis G.B. Ownbey (Papaveraceae). Esta especie se caracteriza por ser una hierba de distribución cosmopolita, nativa en Argentina, de hábito malezoide, cuya presencia y utilización en la zona referida por Montenegro se halla sumamente difundida.

Respecto a los "cardos", la obra del jesuita Florián Paucke refiere propiedades combustibles y forrajeras sin indicar el grupo humano que lo utiliza, en los siguientes términos: "Si escasea la leña, la cocina resulta bastante fría y los viajeros deben de remediarse con cardos secos, gruesas matas de plantas campestres" (Paucke, [1776-78] 1942: 125), y "De las raices de estos cardos se alimentan tanto los puercos silvestres como los caseros de los cuales yo tenía alrededor de trescientos que corrían por la isla [y] de los que escribiré en mejor oportunidad cuando dé noticias del ganado doméstico" (Paucke, [1776-78] 1944: 185). Sin embargo, como resulta frecuente en la adscripción de los usos aborígenes, al describir su empleo como alimenticio se refiere explícitamente que corresponde a "ellos" (es decir a "los mocovíes") en la siguiente cita: "Ellos tienen un cardo que en su mayor parte crece en la isla entre el río Paraná y el río De los dorados a cuya orilla estaba establecida mi reducción del Santo Xaverij [San Javier]. La caña [del cardo] que frecuentemente tiene una altura de dos varas la cortan mientras está aún verde y jugosa y la comen bien sea cruda o también cocida; tiene un olor y sabor balsámico; los mocovies lo llaman apologo" (Paucke, [177678] 1944: 185). Sin embargo, esta entidad no se correspondería a una especie de Compositae, sino más bien a una del género Eryngium (Apiaceae) que recibe el nombre vulgar de "carda" (ver Rosso \& Scarpa, 2012). Por otro lado, en la lámina LIII (Fig. 1A) se ilustran dos plantas florecidas que el autor denomina "cardo hortense" (Fig. 1A: a) y "cardo" (Fig. 1A: b).

Respecto al "azafrán" Paucke ([1776-78] 1944: 206) refiere en el texto de su obra que: "El azafrán paracuario es rojo amarillo, no tiene sabor, da sólo una sopa amarilla, pero sin embargo es sano. Los españoles llaman este azafrán alazor. Él crece en un arbusto de una vara de alto que tiene muchas ramas erectas con muchas hojas verdeobscuras; cada hoja tiene en la punta una púa. Las hojas tienen un largo de un medio dedo y un ancho de una pulgada; en cada rama hay una flor amarilla que tiene abajo un botón; en éste se halla la semilla que es blanca y tiene el tamaño de la semilla del girasol o solsequia. Los españoles llaman a esta flor: Mirasol. La flor se parece a una pequeña flor de cardo, no tiene hojas, sino que sólo brotan cortas hebritas amarillas que se arrancan, se tuestan y se guardan para el uso. La semilla se cuece y el agua proveniente de ahi se bebe; es muy buena para el pecho, lo purifica por la expectoración y saca también la flema por la evacuación". En la lámina XLVI (Fig. 1B) de la misma obra se ilustran en acuarela colorida plantas florecidas con el nombre de "azafrán de Indias"; posiblemente sean tres plantas de la misma especie, de las cuales la primera se muestra ramificada desde la base con tres tallos principales (Fig. 1B, c) y las otras dos con sus tallos no ramificados (Fig. 1B, d).

Respecto al "azafrán”, Lozano ([1733] 1874: 268-269) afirma lo siguiente: "El azafrán que llaman de la tierra, fuera de dar color á las comidas, sirve su zumo de remedio eficaz contra la ictericia". Por su lado, Dobrizhoffer ([1784] 1967: 533) señala que "Igualmente falta en toda Paracuaria el croco, llamado español Azafrán. El [azafrán] americano tiene en común con el nuestro únicamente el nombre y la figura ya se use solo para teñir de amarillo pero no para condimentar las comidas". En relación a lo planteado por Lozano y en coincidencia con Dobrizhoffer, la entidad americana no sería Crocus sativus L. (Iridaceae) conocido como "azafrán", sino que podría referirse a Carthamus tinctorius L. llamado "cártamo" o "falso azafrán", entre otros nombres vulgares.

Cabe destacar que en las obras de Cardiel ([1748] 1930), Concolorcorvo ([1773] 1908) y Sánchez 
Labrador ([1771-1776] 1901) no se hallaron referencias a "cardos" o de estar mencionados dichas entidades ya fueron analizadas por autores previos y se citan en la bibliografía de nuestro trabajo.

Identidad taxonómica del "azafrán alazor", "azafrán de Indias" o "azafrán paracuario"

En relación a la entidad denominada "azafrán alazor", "azafrán de Indias" o "azafrán paracuario", sobre la base de la descripción morfológica e ilustraciones en el trabajo de Paucke (Fig. 1B) se pudieron definir los siguientes caracteres morfológicos: hábito, altura, ramificación del tallo, filotaxis, presencia/ausencia de pecíolo, lámina foliar, ápice foliar, margen foliar, color de las hojas, inflorescencia y número de capítulos, involucro, filaria, número y color de las flores (Tabla 1). Dicha entidad se ajusta morfológicamente al género Carthamus como fuera indicado en trabajos previos.

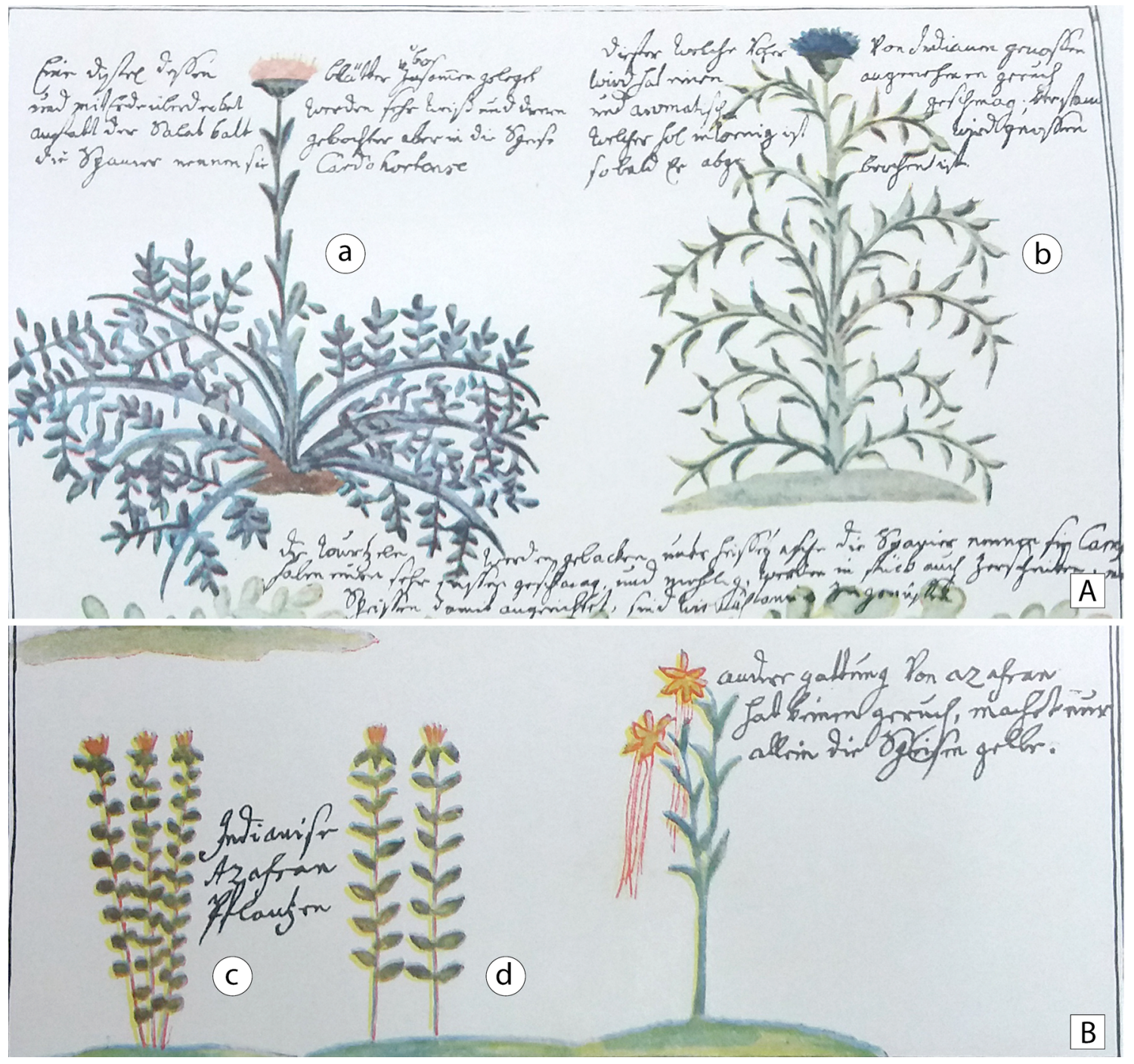

Fig. 1. Azafrán y cardos en la obra de Paucke. A: Parte superior de la lámina LIII con ilustraciones de "cardos". B: Parte inferior de la lámina XLVI con ilustraciones del "azafrán". Abreviaturas = a: "cardo hortense"; b: "cardo"; c y d: "azafrán alazor", azafrán de Indias" o "azafrán paracuario". 


\section{G. Gutiérrez et al. - "Cardos" en Argentina en el siglo XVIII y etnobotánica}

Tabla 1. Caracteres morfológicos del azafrán paracuario, cardo y cardo hortense identificados en las ilustraciones y descripciones escritas de la obra de Paucke.

\begin{tabular}{|c|c|c|c|}
\hline Carácter/Entidad & $\begin{array}{l}\text { Azafrán alazor, azafrán de } \\
\text { Indias o azafrán paracuario }\end{array}$ & Cardo & Cardo hortense \\
\hline Hábito & Herbáceo & Herbáceo & Herbáceo \\
\hline Altura & Ca. $85 \mathrm{~cm}$ & - & - \\
\hline Tallo & $\begin{array}{l}\text { Ramificado desde la base } \\
\text { o no ramificado }\end{array}$ & No ramificado & No ramificado \\
\hline Filotaxis & $\begin{array}{c}\text { Opuesta o subopuesta } \\
\text { distribuida a lo largo del tallo }\end{array}$ & En roseta basal & En roseta basal \\
\hline $\begin{array}{l}\text { Presencia/ausencia } \\
\text { de pecíolo }\end{array}$ & Ausente o inconspicuo & Presente & Presente \\
\hline Lámina foliar & Simple, no dividida & Compuesta o muy dividida & Compuesta o muy dividida \\
\hline Margen foliar & Entero & Dentado & Entero \\
\hline Color de las hojas & Verde & Verde & Celeste \\
\hline Inflorescencia & Terminal, capítulo solitario & $\begin{array}{l}\text { Escaposa con un } \\
\text { capítulo terminar }\end{array}$ & $\begin{array}{l}\text { Escaposa con un } \\
\text { capítulo terminal }\end{array}$ \\
\hline Involucro & - & $\begin{array}{l}\text { Hemisférico o anchamente } \\
\text { acampanado }\end{array}$ & $\begin{array}{c}\text { Hemisférico o anchamente } \\
\text { acampanado }\end{array}$ \\
\hline Filarias & Foliáceas & - & - \\
\hline Número de flores & Numerosas & Numerosas & Numerosas \\
\hline Color de las flores & Anaranjado & Azulado & Amarillo \\
\hline
\end{tabular}

Los caracteres que permiten una adecuada identificación son los siguientes: plantas de entre $30-90 \mathrm{~cm}$ de altura, con tallos erectos, ramificados, las hojas alternas, sésiles, ovadas o elípticas, con ápice agudo y margen entero o aserradoespinoso, los capítulos solitarios en las ramas terminales, y generalmente varios por planta, sésiles o con pedúnculos muy cortos, involucro ovoide con filarios foliáceos con los externos, ovados de ápice agudo y margen entero, denticulado o serrado-espinoso, y las flores numerosas, con corolas amarillas, anaranjadas, rojas o blancas. De esta manera la entidad “azafrán alazor", “azafrán de Indias" o "azafrán paracuario" de Paucke puede identificarse correctamente como Carthamus tinctorius principalmente por presentar la lámina foliar entera y los filarios externos foliáceos y enteros, con las flores anaranjadas o amarilloanaranjadas (Fig. 2A-D). Dicha especie recibe en Argentina el nombre vulgar de "cártamo" o "falso azafrán". Al mismo tiempo se descarta que dicha entidad sea $C$. lanatus (vulgarmente "cardo chileno", "cardo cruz", "cardo del diablo", "cardo lanudo", "cártamo", "manca potrillo") dado que esta especie presenta hojas lobadas de margen espinoso y flores amarillas (Fig. 2E-F).

\section{Identidad taxonómica del "cardo" y "cardo} hortense"

Respecto a las entidades mencionadas en el trabajo de Paucke como "cardo" y "cardo hortense" y a sus correspondientes ilustraciones (Fig. 1A) se pudieron identificar y seleccionar los siguientes caracteres morfológicos: (1) hábito, (2) ramificación del tallo, (3) filotaxis, (4) presencia/ ausencia de pecíolo, (5) lámina foliar, (6) margen foliar, (7) color de las hojas, (8) inflorescencia y número de capítulos, (9) involucro, (10) filaria, (11) número y (12) color de las flores (Tabla 1). Los caracteres que permiten una adecuada identificación son los siguientes: hierbas con hojas basales arrosetadas, pecíolos largos y láminas profundamente pinnatisectas de contorno ovado, con segmentos de ápice atenuado con una espina terminal y margen aserrado-lobulado con dientes terminados también en espina y espinas basales, 
Bol. Soc. Argent. Bot. 55 (2) 2020
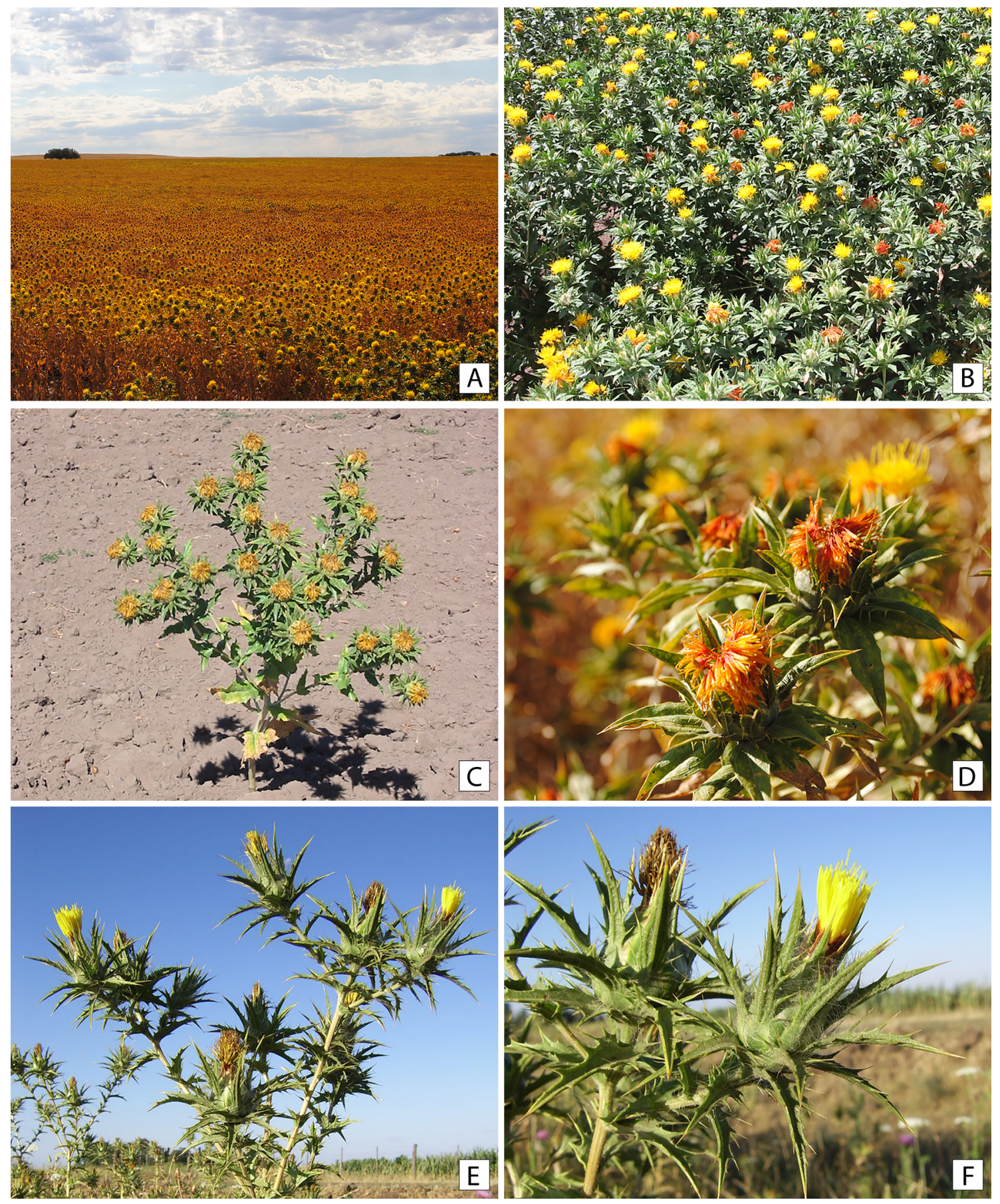

Fig. 2. Carthamus tinctorius y C. lanatus. A-D: Carthamus tinctorius ("cártamo"). A: Cultivo. B: Población. C: Planta. D: Capítulos. E-F: C. lanatus ("cardo lanudo"). E: Parte superior de una planta. F: Capítulos. Fotos: Julio C. Rivas (A-D) en Cardenal Cagliero (prov. Buenos Aires) y Diego G. Gutiérrez (E-F) en Azul (prov. Buenos Aires). 


\section{G. Gutiérrez et al. - "Cardos" en Argentina en el siglo XVIII y etnobotánica}

inermes en las variedades cultivadas, el haz foliar es glauco, y el envés densamente blanco o grisáceo, inflorescencia escaposa con capítulos solitarios con pedúnculos largos, involucro anchamente ovoide o hemisférico y flores numerosas, con corolas generalmente azules o lilas, a veces blancas. De esta manera las entidades "cardo" y "cardo hortense" de Paucke pueden identificarse correctamente ambas como Cynara cardunculus L. (Fig. 3A-F).

Así, "cardo" se asocia a la variedad $C$. cardunculus var. sylvestris (Lam.) Fiori dada la presencia de espinas en hojas y filiarias (Fig. 3AD), y que se denomina vulgarmente en Argentina como "cardo", "cardo de Castilla", "cardo de comer" y "cardón". Por otro lado, el "cardo hortense" por carecer de espinas corresponde a $C$. cardunculus var. scolymus (L.) Fiori (Fig. 3E-F), variedad conocida actualmente como "alcachofa" o "alcaucil".

\section{Nuevos datos etnobotánicos derivados}

En la Tabla 2 se detallan 12 nuevos datos etnobotánicos derivados de las identidades botánicas antes estimadas, indicando para los cuatro taxa involucrados (Carthamus tinctorius, Cynara cardunculus var. sylvestris, C. cardunculus var. scolymus y Argemone subfusiformis) las categorías de los usos registrados, partes utilizadas, aplicación específica y fuente de origen de los mismos. La mayoría de estos datos (cinco) son de índole medicinal y en segunda instancia alimenticios (tres). El taxón sobre el que se registró mayor número de datos fue Cynara cardunculus var. sylvestris con un total de siete datos.

Tabla 2. Datos etnobotánicos de Carthamus tinctorius, Cynara cardunculus var. scolymus, C. cardunculus var. sylvestris y Argemone subfusiformis.

\begin{tabular}{|c|c|c|c|c|c|c|}
\hline $\begin{array}{l}\text { Familia y } \\
\text { especie }\end{array}$ & $\begin{array}{l}\text { Nombres en las } \\
\text { obras de Paucke } \\
\text { y Montenegro }\end{array}$ & $\begin{array}{l}\text { Nombres } \\
\text { vulgares } \\
\text { actuales }\end{array}$ & $\begin{array}{l}\text { Categoría } \\
\text { de uso }\end{array}$ & $\begin{array}{l}\text { Aplicación } \\
\text { específica }\end{array}$ & $\begin{array}{c}\text { Parte } \\
\text { utilizada y } \\
\text { preparación }\end{array}$ & Referencia bibliográfica \\
\hline \multicolumn{7}{|c|}{ Compositae En la obra de Paucke } \\
\hline \multirow{2}{*}{$\begin{array}{l}\text { Carthamus } \\
\text { tinctorius L. }\end{array}$} & \multirow{2}{*}{$\begin{array}{l}\text { "Azafrán alazor", } \\
\text { "azafrán de } \\
\text { Indias", "azafrán } \\
\text { paracuario" }\end{array}$} & \multirow{2}{*}{$\begin{array}{l}\text { "Cártamo", } \\
\text { "falso azafrán" }\end{array}$} & \multirow{2}{*}{$\begin{array}{l}\text { Medicinal } \\
\text { Alimenticio }\end{array}$} & $\begin{array}{l}\text { Contra } \\
\text { ictericia }\end{array}$ & \multirow{2}{*}{$\begin{array}{l}\text { Dec. int. } \\
\text { Flores } \\
\text { tostadas o } \\
\text { cocidas? }\end{array}$} & \multirow{2}{*}{$\begin{array}{c}\text { Lozano ([1733] 1874) } \\
\text { Lozano }([1733] 1874) \text { y } \\
\text { Dobrizhoffer ([1784] 1967) }\end{array}$} \\
\hline & & & & $\begin{array}{l}\text { Colorante } \\
\text { amarillo de } \\
\text { comidas }\end{array}$ & & \\
\hline $\begin{array}{l}\text { Cynara } \\
\text { cardunculus L. } \\
\text { var. scolymus } \\
\text { (L.) Fiori }\end{array}$ & "Cardo hortense" & $\begin{array}{l}\text { "Alcachofa", } \\
\text { "alcaucil" }\end{array}$ & Agricultura & $\begin{array}{l}\text { Cultivado en } \\
\text { la reducción } \\
\text { de San } \\
\text { Javier (pcia. } \\
\text { de Santa Fe) }\end{array}$ & & Paucke ([1776-1778] 1944) \\
\hline \multirow{7}{*}{$\begin{array}{l}\text { Cynara } \\
\text { cardunculus L. } \\
\text { var. sylvestris } \\
\text { (Lam.) Fiori }\end{array}$} & \multirow{7}{*}{ "Cardo" } & $\begin{array}{l}\text { "Cardo", "cardo } \\
\text { de Castilla", }\end{array}$ & Alimenticio & Crudo & Tallos & Paucke ([1776-1778] 1944) \\
\hline & & \multirow{6}{*}{$\begin{array}{l}\text { "cardo de } \\
\text { comer", } \\
\text { "cardón" }\end{array}$} & Alimenticio & Cocidos & Tallos & Paucke ([1776-1778] 1944) \\
\hline & & & Medicinal & Galactogogo & $\begin{array}{l}\text { Dec. int. de su } \\
\text { parte aérea }\end{array}$ & Paucke ([1776-1778] 1944) \\
\hline & & & Medicinal & Expectorante & $\begin{array}{l}\text { Dec. int. de su } \\
\text { parte aérea }\end{array}$ & Paucke ([1776-1778] 1944) \\
\hline & & & Combustible & Leña & Parte aérea & Paucke ([1776-1778] 1944) \\
\hline & & & Forraje & Porcinos & Raíces & Paucke ([1776-1778] 1944) \\
\hline & & & Fauna & $\begin{array}{l}\text { Alimento de } \\
\text { jabalíes }\end{array}$ & Raíces & Paucke ([1776-1778] 1944) \\
\hline \multicolumn{7}{|c|}{ Papaveraceae En la obra de Montenegro } \\
\hline \multirow{2}{*}{$\begin{array}{l}\text { Argemone } \\
\text { subfusiformis } \\
\text { G.B. Ownbey }\end{array}$} & \multirow{2}{*}{ "Cardo santo" } & \multirow{2}{*}{ "Cardo santo" } & Medicinal & $\begin{array}{l}\text { Contra } \\
\text { cefalalgias }\end{array}$ & Parte aérea? & $\begin{array}{c}\text { Montenegro ([1710] 2007); } \\
\text { Lozano ([1733] 1874) y } \\
\text { Dobrizhoffer ([1784] 1967) }\end{array}$ \\
\hline & & & Medicinal & $\begin{array}{l}\text { Contra } \\
\text { amnesia }\end{array}$ & Parte aérea? & $\begin{array}{c}\text { Montenegro ([1710] 2007); } \\
\text { Lozano ([1733] 1874) y } \\
\text { Dobrizhoffer ([1784] 1967) }\end{array}$ \\
\hline
\end{tabular}


Bol. Soc. Argent. Bot. 55 (2) 2020
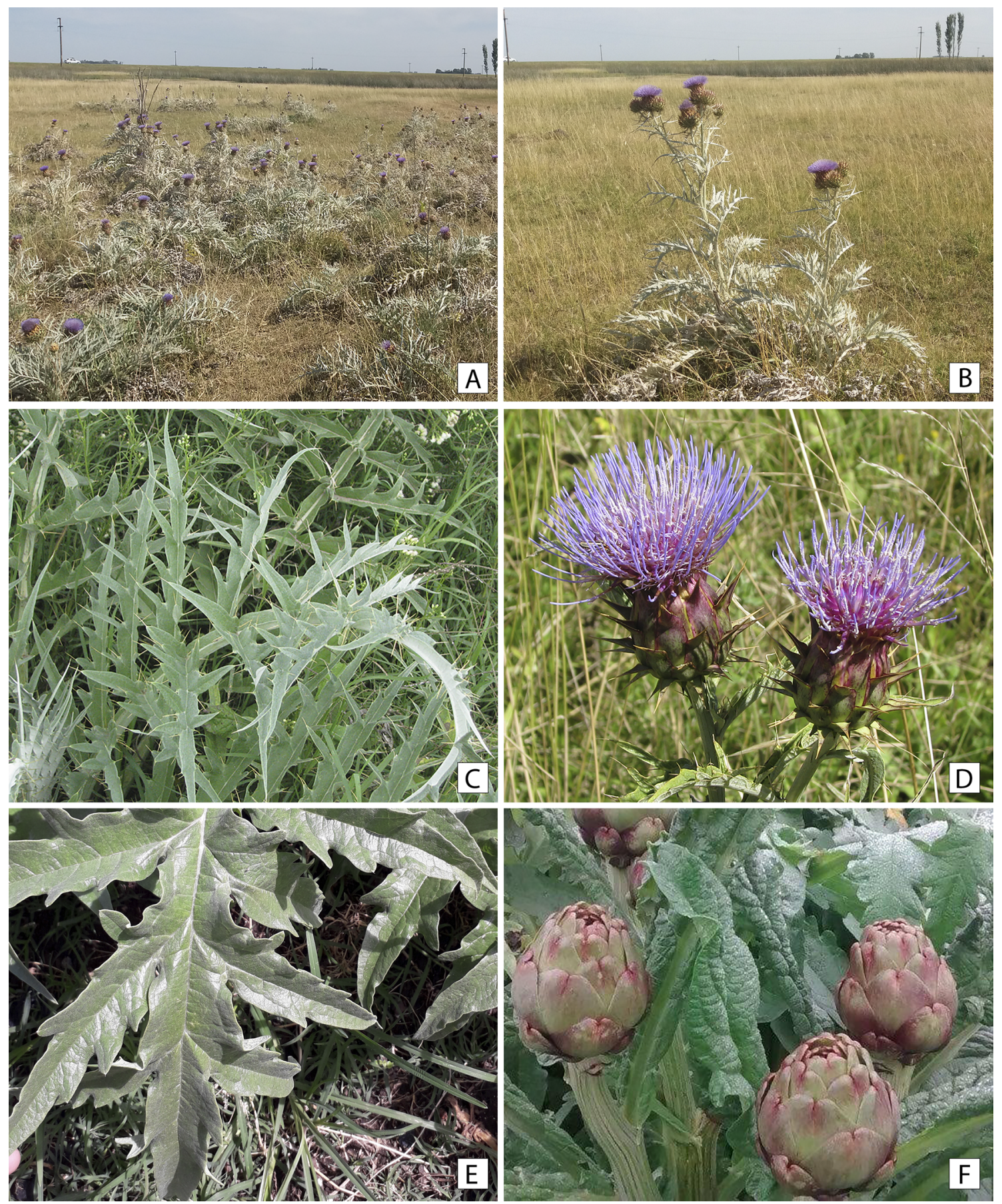

Fig. 3. Cynara cardunculus. A-D: Cynara cardunculus var. sylvestris ("cardo de Castilla"). A: Población. B: Planta. C: Hoja. D: Capítulos. E-F: C. cardunculus var. scolymus ("alcaucil"). E: Parte apical de una hoja. F: Capítulos. Fotos: Diego G. Gutiérrez (A-D) en Azul (prov. Buenos Aires), Vanesa Perrotta (E) en La Plata (prov. Buenos Aires) y María Cristina Mondino (F) en Rosario (prov. Santa Fe). 


\section{G. Gutiérrez et al. - "Cardos" en Argentina en el siglo XVIII y etnobotánica}

\section{Discusión y Conclusiones}

Los datos sobre el cultivo y utilización de los "cardos" mencionados e ilustrados por Paucke podrían ser interpretados a la luz de lo que se conoce como "etnobotánica misionera", según la fórmula concebida por Scarpa \& Anconatani (2017). Esto es, la concepción que sobre dichas plantas poseían los misioneros jesuitas en ese entonces, así como de su cultivo. En efecto, los criterios empleados en la elección de los cultivos utilizados en las parcelas agrícolas de las reducciones aborígenes administradas por los religiosos, respondían básicamente a abastecer de alimentos y otros recursos básicos reputados como "necesarios" para los nativos. Sin embargo, su selección dependía de que a los pueblos originarios les satisficieran, a fin de tornar sustentable no solo la práctica misma de la agricultura (inédita para pueblos cazadores-recolectores como los moqoit de Paucke), sino sobre todo como acicate para propiciar que los aborígenes no abandonaran la reducción y volvieran a internarse en lo profundo del bosque chaqueño. Incluso, los jesuitas comenzaron a plantar especies silvestres que los nativos utilizaban tradicionalmente en su alimentación como forma de promover que estos se mantuvieran dentro del espacio misional. Un ejemplo de ello es que Paucke llegó a cultivar Cynophalla retusa (Griseb.) Cornejo \& Iltis (Capparaceae; "poroto del monte", "fasoles") con el objetivo de que espaciaran sus prácticas de caza-recolección (Rosso, 2012).

La conceptualización de los misioneros jesuitas (e.g. Paucke y Montenegro) sobre la categoría "cardo" incluía toda planta herbácea con espinas, tanto a las especies exóticas introducidas (e.g. Cynara cardunculus) como a las americanas pertenecientes a las familias Bromeliaceae y Apiaceae del género Eryngium, tal como explícitamente lo refieren estudios previos (Rosso \& Scarpa, 2012). Esto último, sumado a la gran significación que las especies de Bromelia tenían en todas las culturas aborígenes del Chaco (incluida la moqoit) como recurso textil y alimenticio, conjuntamente a que el consumo de sus bases foliares carnosas (al igual que la de los "alcauciles") constituyen un ítem muy valorado de su culinaria tradicional (Rosso \& Scarpa, 2017), permitiría inferir que estos podrían haber sido seleccionados para su cultivo a los fines de satisfacer en las mismas misiones la necesidad de los pueblos originarios de abastecerse de los "cardos salvajes" en el monte chaqueño.

Respecto al trabajo de Montenegro ([1710] 2007) resulta muy sugerente que ningún "cardo" figure en el cuerpo principal de la obra donde cada una de las plantas son tratadas e ilustradas en detalle y solo se mencionen muy brevemente hacia el final de la misma. Debido a que está demostrado que esta obra sufrió agregados que resultan posteriores a 1750 , por su ubicación hacia el final del texto y por no responder a la estructura principal del manuscrito, estimamos que la temporalidad de dichas citas sería la misma que los añadidos (Arata, 1898; Scarpa \& Anconatani, 2019). En relación a la entidad mencionada en esta obra como "cardo santo" y en nuestro trabajo indentificada como Argemone subfusiformis, dicha asociación reforzaría citas antiguas posteriores sobre registro y usos sudamericanos de especies del género Argemone (Azara, 1809). Argemone subfusiformis es una especie cosmopolita y presenta una amplia distribución en América del Sur alcanzando el centro de la Patagonia (Ownbey, 1961; PérezMoreau, 1984; Petenatti \& Del Vitto, 2001; Zuloaga et al., 2008). Esta especie se caracteriza por ser maleza (Marzocca, 1986; Fernández et al., 2016) con un registro de presencia y utilización en la zona referida por Montenegro sumamente difundidos (Hieronymus, 1882; Sorarú, 1976; Toursarkissian, 1980; Barboza et al., 2006; Scarpa \& Anconatani, 2019).

Sobre el "cardo" de Paucke identificado como Cynara cardunculus var. sylvestris ("cardo de Castilla"), cabe destacar que la especie $C$. cardunculus es originaria del Mediterráneo y Macronesia en ambientes costeros, habiéndose naturalizado en Australia, México, el estado de California de Estados Unidos, y en América del Sur en Chile, Uruguay y Argentina (Wiklund, 1992; Randall, 2007; Delucchi, 2008; Leak-Garcia et al., 2012; Gutiérrez \& Sonnante, 2014), actualmente muy extendida como maleza (Marzocca, 1986; Fernández et al., 2016). El "cardo hortense" de Paucke, que corresponde a $C$. cardunculus var. scolymus ("alcaucil") es utilizada a nivel mundial como una especie alimenticia, industrial, medicinal y ornamental desde el Imperio Romano (Sonnante $e t$ $a l ., 2007)$ y su cultivo se encuentra muy difundido, siendo algunos países sudamericanos (i.e. 
Argentina, Chile y Perú) importantes productores mundiales (Calabrese, 2016).

Respecto a la nueva identificación del "azafrán paracuario" de Paucke como la especie Carthamus tinctorius ("cártamo" o "falso azafrán"), las implicancias históricas en relación al uso e introducción de especies del Viejo Mundo son muy importantes. C. tinctorius es una especie originaria de la región Irano-turánica (oeste de Asia) que se ha extendido de forma secundaria a diferentes partes del mundo (Zhou et al., 2014). A diferencia de otras Cardueas introducidas, probablemente pueda solo considerarse como adventicia ocasional, escapada de cultivo, en el noroeste y centro-este de Argentina (Delucchi, 2002; Gutiérrez \& Vilatersana, 2014).

Asociadas a las identificaciones botánicas estimadas a lo largo de este trabajo se registran varios nuevos datos etnobotánicos. Entre estos destacan los usos medicinales (cinco usos) y los alimenticios (tres usos). Estos últimos revisten especial interés dado que constituye el objeto mismo por el cual se habrían introducido las variedades de Cynara cardunculus en América del Sur y su cultivo, registrado aquí en la misión San Javier. De esta manera se confirma que los "cardos" nativos o introducidos fueron utilizados con fines medicinales y alimenticios durante el periodo colonial, y en particular las especies introducidas fueron rápidamente adoptadas por los pueblos aborígenes. En el caso de Cynara cardunculus y sus variedades ("alcaucil" y "cardo de Castilla") y Carthamus tinctorius ("cártamo"), taxones actualmente cultivados, naturalizados o adventicios en el sur de América del Sur, nuestro trabajo confirmaría el planteo seguido por varios autores según los cuales sus ingresos a la región habrían sido como especies hortícolas, siendo las primeras Asteráceas introducidas, registradas mediante ilustraciones, para mediados el siglo XVIII en Argentina.

\section{Contribución de los AUtORES}

DGG concibió la presente investigación. DGG diseñó y dirigió el estudio sistemático y morfológico; GFS y CNR diseñaron y dirigieron el estudio etnobotánico y llevaron a cabo el análisis de la bibliografía etnobotánica y de cronistas históricos. Todos los autores intervinieron mediante una interacción crítica y trabajaron colaborativamente en la investigación, análisis y redacción del manuscrito.

\section{Agradecimientos}

Los autores agradecen a Verónica Lema (Instituto de Humanidades, CCT-Córdoba-CONICET) por la lectura crítica del manuscrito que ayudaron a mejorarlo notablemente. Al personal de los herbarios BA, BAA, BAB, BAF, CORD, CTES, LP, LPAG, MERL, SI y UNR por la asistencia técnica y facilitarnos materiales o fotografías para llevar a cabo este trabajo. A Mariana Grossi, Gonzalo Marquez, Juan Rodríguez-Cravero, Miguel Quijano, Jessica Viera-Barreto y Agustina Yañez y Vanina Salgado por su ayuda en los viajes de campo. A María Cristina Mondino (FCA-UNR e INTA), Vanesa Perrotta (FCAyF-UNLP) y Julio Rivas (INTA) por facilitarnos las fotografías de Carthamus tinctorius y Cynara cardunculus var. scolymus. El trabajo fue financiado por el Consejo Nacional de Investigaciones Científicas y Técnicas (CONICET; PUE 22920160100098CO), y en el caso de D.G.G. además por la Agencia Nacional de Promoción Científica y Tecnológica (PICT-2017-0965) y la Universidad Nacional de La Plata (UNLP N814).

\section{Biblografía}

AMARAL, S. 1997. Producción agropecuaria 18101850. Academia Nacional de la Historia, Nueva Historia de la Nación 6: 41-64. Ed. Planeta, Buenos Aires.

AMARAL, S. 1999. Auge y decadencia del cardo en el siglo XIX. Anales de la Sociedad Rural Argentina 123: 42-47.

ARATA, P.N. 1898. Botánica médica americana. Los herbarios de las misiones del Paraguay. La Biblioteca 22: 419-448.

ARIZA ESPINAR, L. \& G. DELUCCHI. 1998. Tribu Cardueae. Flora Fanerogámica Argentina 60: 1-26.

AVILA, F., FUNK, V.A., DIAZGRANADOS, M., DÍAZPIEDRAHITA, S. \& VARGAS, O. 2016. Asteraceae. En: BERNAL, R., S. ROBBER GRADSTEIN \& M. CELIS (eds.), Catálogo de plantas y líquenes de Colombia, vol. 1. Universidad Nacional de Colombia, Bogotá, pp. 795-908. 


\section{G. Gutiérrez et al. - "Cardos" en Argentina en el siglo XVIII y etnobotánica}

AZARA, F. DE, 1809. Voyages dans l'Amérique Méridionale. T. 1. Dentu, París. https://doi.org/10.5962/bhl.title.84598

BADILLO, V.M. 2001. Lista actualizada de las especies de la familia Compuestas (Asteraceae) de Venezuela. Ernstia 11: 147-215.

BAKER, J.G. 1876. Subordo IX. Cynaroideae (Compositae). En: MARTIUS, C.F.P. VON (ed.), Flora Brasiliensis, vol. 6(3): 325-330. F. Fleischer, Monachii \& Lipsiae.

BARBOZA, G.E., J.J. CANTERO, C.O. NUÑEZ \& L. ARIZA ESPINAR. 2006. Flora medicinal de la Provincia de Córdoba (Argentina). Pteridófitas y Antófitas silvestres y naturalizadas. Museo Botánico, Córdoba.

BECK, S.G., D. IBAÑEZ, P.M. JØRGENSEN, J. MÜLlER, J.E. PRUSKY, H. ROBINSON, E. URTUBEY, K. TREMETSBERGER \& T. STUESSY. 2014. Asteraceae. En: JØRGENSEN, P.M., M.H. NEE \& S.G. BECK (eds.), Catálogo de las plantas vasculares de Bolivia. Monogr. Syst. Bot. Missouri Bot. Gard. 127: 290-382.

BERG, C. [F.W.K.]. 1877. Enumeración de las plantas europeas que se hallan como silvestres en la provincia de Buenos Aires y en Patagonia. Pablo E. Coni, Buenos Aires. https://doi.org/10.5962/bhl.title.9301

CABRERA, A.L. \& A. WILLINK. 1980. Biogeografia de América Latina, 2nd ed. Serie de Biología, Monografía 13. Organización de los Estados Americanos, Washington.

CALABRESE, N. 2016. From Mediterranean basin to the Andean mountains, the long journey of the artichoke that unites different people and cultures. ISHS Acta Horticulturae 1147: 23-34. https://doi.org/10.17660/ActaHortic.2016.1147.4

CAPPARELLI, A., V. LEMA, M. GIOVANETTI \& R. RAFFINO. 2005. The introduction of Old World crops (wheat, barley and peach) in Andean Argentina during the 16th century a.d.: archaeobotanical and ethnohistorical evidence. Veget. Hist. Archaeobot. 14:472-484.

https://doi.org/10.1007/s00334-005-0093-8

CARDIEL, J. [1748] 1930. Diario de viaje y misión al río Sauce en 1748 por el p. José Cardiel, sj, con un estudio biográfico del autor por G. Furlong, sj. Imprenta Coni, Buenos Aires.

CONCOLORCORVO. [1773] 1908. El lazarillo de ciegos caminantes desde Buenos Aires hasta Lima. 1773. Junta de Historia y Numismática Americana. Cía. Sudamericana de Billetes de Banco. Buenos Aires.
CORDERO, S., D.G. GUTIÉRREZ \& A. MOREIRA. 2016. Carduus tenuiflorus Curtis (Asteraceae), nuevo registro para la flora alóctona asilvestrada de Chile. Gayana, Botánica 73: 436-439. https://doi.org/10.4067/S0717-66432016000200453

CORREA, R.F., G. DELUCCHI \& G. PARRA. 2003. Etnobotánica de los "cardos" en la Argentina. Delpinoa 45: 149-156.

DARWIN, C. R. 1839. Narrative of the surveying voyages of His Majesty's Ships Adventure and Beagle between the years 1826 and 1836, describing their examination of the southern shores of South America, and the Beagle's circumnavigation of the globe. Journal and remarks. 1832-1836. Henry Colburn, Londres.

DELUCCHI, G. 2002. Sobre la presencia de una nueva especie adventicia de Carthamus (Asteraceae, Cardueae) en la Argentina. Hickenia 3: 129-131.

DELUCCHI, G. 2008. Cardueae (Asteraceae). En: ZULOAGA, F.O., O. MORRONE \& M.J. BELGRANO (eds.), Catálogo de las Plantas Vasculares del Cono Sur (Argentina, Sur de Brasil, Chile, Paraguay y Uruguay). Monogr. Syst. Bot. Missouri Bot. Gard. 107. Asteraceae vol. 2: 11541565.

DELUCCHI, G., F. CORREA \& G. CHARRA. 2003. Génesis del complejo de los "cardos" en la Región Pampeana (Argentina). Delpinoa 45: 143-148.

DELUCCHI, G. \& D.G. GUTIÉRREZ. 2014. Tribu Cardueae Cass. (Asteraceae). En: ZULOAGA, F.O., M.J. BELGRANO \& A.M.R. ANTON (eds.), Flora Argentina, Flora Vascular de la República Argentina. Dicotyledoneae, Asteraceae, 7: 271-296. Estudio Sigma S.R.L., Buenos Aires.

DELUCCHI, G., D.G. GUTIÉRREZ \& J.A. HURRELL. 2017. Tribu Cardueae Cass. (Asteraceae). En: HURRELL, J.A., N.D. BAYÓN \& G. DELUCCHI (eds.), Plantas Cultivadas de la Argentina. Asteráceas (= Compuestas). Editorial Hemisferio Sur S.A., Buenos Aires.

DILLON, M.O. 2006. Nuevos registros de Asteraceae para la flora de Perú. Arnaldoa 13: 314-317.

DILLON, M.O. \& N. HENSOLD. 1993. Asteraceae. En: BRAKO, L. \& J.L. ZARUCCHI (eds.), Catalogue of the Flowering Plants and Gymnosperms of Peru. Monogr. Syst. Bot. Missouri Bot. Gard. 45: 103-189.

DOBRIZHOFFER, M. [1784] 1967. Historia de los abipones. Vol. 1. Universidad Nacional del Nordeste, Resistencia. 
EKIN, Z. 2005. Resurgens of Safflower (Carthamus tinctorius L.) utilization: a global view. J. Agron. 4: 83-87. https://doi.org/10.3923/ja.2005.83.87

FERNÁNDEZ, O.A., E.S. LEGUIZAMÓN \& H.A. ACCIARESI. 2016. Malezas e Invasoras de la Argentina, Tomo II: Descripción y reconocimiento. Universidad Nacional del Sur, Bahía Blanca.

FONT QUER, P. 1993. Diccionario de botánica. $1^{\mathrm{a}}$ edición, $11^{\mathrm{a}}$ reimpresión. Labor, Barcelona.

GATTO, A., D. DE PAOLA, F. BAGNOLI, G.G. VENDRAMIN \& G. SONNANTE. 2013. Population structure of Cynara cardunculus complex and the origin of the conspecific crops artichoke and cardoon. Ann. Bot. 112: 855-865.

https://doi.org/10.1093/aob/mct150

GOMINHO, J., A. LOURENÇO, M. CURT, J. FERNÁNDEZ \& H. PEREIRA. 2009. Characterization of hairs and pappi from Cynara cardunculus capitula and their suitability for paper production. Ind. Crop. Prod. 29: 116-125. https://doi.org/10.1016/j.indcrop.2008.04.022

GRISEBACH, A. 1879. Symbolae ad floram argentinam. Abh. Königl. Ges. Wiss. Göttingen 24: 1-345.

GRUZINSKI, S. 2007. El pensamiento mestizo. Cultura amerindia y civilización del Renacimiento. Paidós, Barcelona.

GUTIÉRREZ D.G \& G. SONNANTE. 2014. Cynara. En: ZULOAGA, F.O., M.J. BELGRANO \& A.M.R. ANTON (eds.), Flora Argentina, Flora Vascular de la República Argentina. Dicotyledoneae, Asteraceae, 7: 290-291. Estudio Sigma S.R.L., Buenos Aires.

GUTIÉRREZ D.G \& R. VILATERSANA. 2014. Carthamus. En:ZULOAGA, F.O., M.J.BELGRANO \& A.M.R. ANTON (eds.), Flora Argentina, Flora Vascular de la República Argentina. Dicotyledoneae, Asteraceae, 7(1): 277-279. Estudio Sigma S.R.L., Buenos Aires.

HEAD, F.B. 1846. Rough notes taken during some rapid journeys across the Pampas and among the Andes. William Clowes and sons, Londres.

HERNÁNDEZ BERMEJO, J.E., G. DELUCCHI, G. CHARRA, M.L. POCHETTINO \& J.A. HURRELL. 2019. "Cardos" of two worlds: transfer and resignification of the uses of thistles between the Iberian peninsula and Argentina. Ethnobiol. Conserv. 8: 5. https://doi.org/10.15451/ec2019-03-8.05-1-22.

HIERONYMUS, J. 1882. Plantae diaphoricae Florae Argentinae. Guillermo Kraft, Buenos Aires.

HILGERT, N.I., D.A. LAMBARÉ, N.D. VIGNALE, P.C. STAMPELLA \& M.L. POCHETTINO. 2014.
¿Especies naturalizadas o antropizadas? Apropiación local y la construcción de saberes sobre los frutales introducidos en época histórica en el norte de Argentina. Rev. Biodivers. Neotrop. 4: 69-87. https://doi.org/10.18636/bioneotropical.v4i2.118

HOOKER, W.J. \& G.A.W.ARNOTT. 1835. Contributions towards flora of South America and the islands of the pacific. Companion Bot. Mag. 1: 102-111.

KATINAS, L., D.G. GUTIÉRREZ, M.A. GROSSI \& J.V. CRISCI. 2007. Panorama de la familia Asteraceae (Compositae) en la República Argentina. Bol. Soc. Argent. Bot 42: 113-129.

LEAK-GARCIA, A., J.S. HOLT, S.-C. KIM, L. MU, J.A. MEJÍAS \& N.C. ELLSTRAND. 2012. More than multiple introductions: Multiple taxa contribute to the genesis of the invasive California's wild artichoke thistle. J. Syst. Evol. 51: 295-307. https://doi.org/10.1111/j.1759-6831.2012.00222.x

LOZANO, P. [1733] 1874. Historia de la conquista del Paraguay, Río de la Plata y Tucumán.

MARZOCCA, A. 1986. Manual de malezas. $3^{\mathrm{a}}$ edición. Editorial Hemisferio Sur, Buenos Aires.

MONTENEGRO, P. de. [1710] 2007. Materia médica misionera. Editorial Universitaria de Misiones, Posadas.

OWNBEY, G.B. 1961. The genus Argemone in South America and Hawaii. Britonia 13: 91-109. https://doi.org/10.2307/2805287

OYARZABAL, M., J. CLAVIJO, L. OAKLEY, F. BIGANZOLI, P. TOGNETTI, I. BARBERIS, H.M. MATURO, R. ARAGÓN, P.I. CAMPANELLO, D. PRADO, M. OESTERHELD \& R.J.C. LEÓN. 2018. Unidades de vegetación de la Argentina. Ecol. Austral 28: 40-63. https://doi.org/10.25260/EA.18.28.1.0.399

PAUCKE, F. [1776-1778] 1942-1944. Hacia allá y para acá. Una estadía entre los indios mocobies, 1749-1767. Tomos 1-3. Universidad Nacional de Tucumán, San Miguel de Tucumán.

PEÑA, M.R. DE LA \& J.F. PENSIERO. 2004. Plantas argentinas. Catálogo de nombres comunes. L.O.L.A., Buenos Aires.

PÉREZ-MOREAU, R.M. 1984. Papaveraceae. En: CORREA, M.N. (ed.), Flora Patagónica 8(4a): 359-367.

PETENATTI, E.M. \& L.A. DEL VITTO. 2001. Papaveraceae. Fl. Fanerog. Argent. 77: 1-12.

RANDALL, R.P. 2007. The introduced flora of Australia and its weed status. CRC for Australian Weed Managemen, Adelaide. 


\section{G. Gutiérrez et al. - "Cardos" en Argentina en el siglo XVIII y etnobotánica}

RECALT, G. 2004. Los cardos y las tareas rurales en la provincia de Buenos Aires durante el siglo XIX. Mundo Agrario Revista de Estudios Rurales 4(8). https://www.mundoagrario.unlp.edu.ar/issue/view/ v04n08

RÉMY, E.J. 1849. Compuestas. En: GAY, C. (ed.), Historia física y política de Chile. Botánica, t. 4. C. Gay, París. Pp: 1-317.

RIVERA, D. \& C. OBÓN. 1996. Palaeoethnobotany of Compositae in Europe, North Africa and Near East. En: CALIGARI, P.D.S. \& D.J.N. HIND (eds.), Compositae: biology and utilization. Royal Botanic Gardens, Kew.

ROBBA, L., M.A. CARINE, S.J. RUSSELL \& F.M. RAIMONDO. 2005. The monophyly and evolution of Cynara L. (Asteraceae) sensu lato: evidence from the Internal Transcribed Spacer region of nrDNA. Pl. Syst. Evol. 253: 53-64. https://doi.org/10.1007/s00606-004-0259-3

ROBINSON, H., B. NORDENSTAM, R. LUNDIN \& P.M. JØRGENSEN. 1999. Asteraceae. En: JØRGENSEN, P.M. \& S. LEÓN-YÁÑEZ (eds.). Catalogue of the Vascular Plants of Ecuador. Monogr. Syst. Bot. Missouri Bot. Gard. 75: 260-310.

ROIG, F.A. 2001. Flora medicinal mendocina. EDIUNC, Mendoza.

ROSSO, C.N. 2012. La etnobotánica de los grupos mocovies de la reducción de San Javier, en el Gran Chaco durante el siglo XVIII. Tesis Doctoral, Facultad de Filosofía y Letras, Universidad de Buenos Aires.

ROSSO, C.N. \& G.F. SCARPA. 2012. Identificaciones botánicas de las plantas empleadas entre los mocovíes en la reducción de San Javier durante el siglo XVIII a partir de la obra de Florián Paucke, S.J. En ARENAS, P. (ed.), Etnobotánica en zonas áridas y semiáridas del Cono Sur de Sudamérica. Edición CEFYBO-CONICET, Buenos Aires.

ROSSO, C.N. \& G.F. SCARPA. 2017. Etnobotánica de la alimentación entre los indígenas moqoit actuales de la provincia del Chaco (Argentina) y comparación con fuentes históricas de los siglos XVIII y XX. Bol. Soc. Argent. Bot. 52: 827-840. https://doi.org/10.31055/1851.2372.v52.n4.18874

SÁNCHEZ LABRADOR, J. [1771-1776] 1901. El Paraguay católico. Imprenta de Coni Hermanos, Buenos Aires.

SCARPA, G.F. \& L.M. ANCONATANI. 2017. Etnobotánica histórica de las Misiones Franciscanas del este de Formosa II: Identificación y análisis de datos inéditos y reelaboración integral de fuentes ya publicadas a partir de hallazgos documentales. Dominguezia 33: 37-79.

http://hdl.handle.net/11336/48890

SCARPA, G.F. \& L.M. ANCONATANI. 2019. La

"Materia Médica Misionera" atribuida al jesuita Pedro de Montenegro en 1710: Identificación, sistematización e interpretación de los usos medicinales de las plantas y sus implicancias para la etnobotánica actual. IHS. Antiguos Jesuitas en Iberoamérica 7: 27-46.

http://dx.doi.org/10.31057/2314.3908.v7.n1.24771

SCHULZ, A.G. 1976. Nombres comunes de las plantas. Talleres Gráficos Moro Hnos., Resistencia.

SIMPSON, B.B. 2009. Economic importance of Compositae. En: FUNK, V.A., A. SUSANNA, T. STUESSY \& R.J. BAYER (eds.), Systematics, evolution, and biogeography of Compositae. International Association of Plant Taxonomists (IAPT), Viena.

SORARÚ, S.B. 1976. Nota sobre el género Argemone (Papaveraceae) en la República Argentina. Darwiniana 20: 445-457.

SONNANTE, G., D. PIGNONE \& K. HAMMER. 2007. The domestication of artichoke and cardoon: From Roman times to the genomic age. Ann. Bot. 100: 1095-1100. https://doi.org/10.1093/aob/mcm127

SORIA, J.L. 2012. Hombres y ganado. La construcción social del pastizal pampeano. 1750-1820. Anuario IEHS 27: 307-320.

STAMPELLA, P.C. 2015. Historia local de naranja amarga (Citrus $x$ aurantium L.) del Viejo Mundo asilvestrada en el corredor de las antiguas Misiones Jesuiticas de la provincia de Misiones (Argentina). Tesis doctoral. Universidad Nacional de La Plata, La Plata.

SUSANNA, A. \& N. GARCIA-JACAS. 2007. Cardueae. En: KADEREIT, J.W. \& C. JEFFREY (eds.), The Families and genera of vascular plants, Flowering Plants, Eudicots, Asterales, vol. 8. Springer, Berlín.

SUSANNA, A. \& N. GARCIA-JACAS. 2009. Cardueae (Carduoideae). En: FUNK, V.A., A. SUSANNA, T. STUESSY \& R.J. BAYER (eds.), Systematics, evolution, and biogeography of Compositae. International Association of Plant Taxonomists (IAPT), Viena.

TOURSARKISSIAN, M. 1980. Plantas medicinales de la Argentina. Editorial Hemisferio Sur S.A., Buenos Aires. 
WERNICKE, E. 1944. Introducción por el traductor. En: PAUCKE, F. [1776-1778] 1944, Hacia allá y para acá. Una estadía entre los indios mocobies, 17491767. Tomo 3. Universidad Nacional de Tucumán, San Miguel de Tucumán.

WIKLUND, A. 1992. The genus Cynara L. (AsteraceaeCardueae). Bot. J. Linnean Soc. 109: 75-123. https://doi.org/10.1111/j.1095-8339.1992.tb00260.x

ZANETTI, S. 2013. Las memorias de Florián Paucke: Una crónica singular de las misiones jesuitas del Gran Chaco Argentino. América Sin Nombre 18: 178189. https://doi.org/10.14198/AMESN2013.18.15
ZHOU, X., L. TANG, Y. XU, G. ZHOU \& Z. WANG. 2014. Towards a better understanding of medicinal uses of Carthamus tinctorius L. in traditional Chinese medicine: a phytochemical and pharmacological review. J. Ethnopharmacol. 151: 27-43. https://doi.org/10.1016/j.jep.2013.10.050 ZULOAGA, F.O., O. MORRONE \& M.J. BELGRANO. 2008. Papaveraceae. Catálogo de las Plantas Vasculares del Cono Sur (Argentina, Sur de Brasil, Chile, Paraguay y Uruguay). Monogr. Syst. Bot. Missouri Bot. Gard. 107(3): 2694-2696. 